\title{
Association of chronotype as assessed by the midpoint of sleep with the dietary intake and health-related quality of life for elderly Japanese women
}

\author{
Natsuko Mito ${ }^{1}$, Eka Fujimoto ${ }^{2}$, Satoshi Sasaki ${ }^{3}$ and the Three-generation Study of Women on Diets and \\ Health Study Group \\ ${ }^{1}$ College of Education, Yokohama National University, Kanagawa, Japan \\ ${ }^{2}$ Department of Food and Nutrition, Faculty of Human Sciences and Design, Japan Women's University, Tokyo, Japan \\ ${ }^{3}$ Department of Social and Preventive Epidemiology, School of Public Health, The University of Tokyo, Tokyo, Japan
}

(Received 31 August 2020 - Final revision received 26 February 2021 - Accepted 17 March 2021)

Journal of Nutritional Science (2021), vol. 10, e25, page 1 of 9

doi:10.1017/jns.2021.16

Abstract

The relationship of chronotype differences with dietary habits and health-related outcomes among elderly people is not fully understood, although sex and generation differences are observed in human chronotype. Accordingly, we analysed the association of chronotype (as assessed by the midpoint of sleep) with dietary intake and health-related quality of life (HRQoL) in elderly Japanese women. The subjects in this cross-sectional study were 1618 women aged 65 years and older who were grandmothers or acquaintances of dietetics students. The subjects were classified into quintiles with respect to the midpoint of sleep, from the earliest to the latest quintile. HRQoL was assessed by the Japanese version of the short-form 36-item health survey score. Mental health was assessed by the Center for Epidemiologic Studies Depression (CES-D) Scale. Dietary intake was assessed by a brief-type self-administered diet history questionnaire. A later midpoint of sleep was associated with a lower intake of vitamin D and a higher intake of bread and caffeinated drinks. No correlations were observed between chronotype and other nutrient and food intake. The subjects with a late midpoint of sleep (eveningness) showed poor general health perception $(\mathrm{GH})$ and high CES-D scores. Other HRQoL scores did not differ among groups with different midpoints of sleep. In conclusion, chronotype as assessed by the midpoint of sleep is associated with poor GH and depressive tendencies in elderly Japanese women. Additionally, a few associations were found between dietary intake and chronotype in elderly Japanese women.

Key words: Chronotype: Dietary intake: Elderly Japanese women: Health-related quality of life: Midpoint of sleep

Many studies suggest that short or long sleep duration, evening chronotype and shift work are related to body weight gain, diabetes, obesity and related chronic diseases in adults and children $^{(1-7)}$. A recent UK cohort study showed that adults aged 38-73 years with a self-described evening chronotype had an increased risk of morbidity and mortality ${ }^{(8)}$. Furthermore, recent reviews have shown an association between dietary intake and chronotype ${ }^{(9,10)}$. Overall, the review showed that evening-type adults mostly engage in unhealthy dietary habits ${ }^{(9)}$. Another review revealed that an evening chronotype is associated with lower intake of fruits and vegetables and higher intake of energy drinks, alcoholic, sugary, caffeinated beverages and energy from fat in adolescents and adults ${ }^{(10)}$. We also reported that a late chronotype as assessed by the Morningness-Eveningness Questionnaire score and midpoint of sleep is associated with poor dietary intake in young Japanese women ${ }^{(11,12)}$. However, there are few studies of chronotype and dietary intake in elderly people.

Abbreviations: CES-D: Center for Epidemiologic Studies Depression; BDHQ: brief-type self-administered diet history questionnaire; HRQoL: health-related quality of life; MET: metabolic equivalent; PSQI: Pittsburgh Sleep Quality Index; SF-36: short-form 36-item health survey

* Corresponding author: Natsuko Mito, email mito-natsuko-jx@ynu.ac.jp

(C) The Author(s), 2021. Published by Cambridge University Press on behalf of The Nutrition Society. This is an Open Access article, distributed under the terms of the Creative Commons Attribution-NonCommercial-NoDerivatives licence (http://creativecommons.org/licenses/by-nc-nd/4.0/), which permits non-commercial re-use, distribution, and reproduction in any medium, provided the original work is unaltered and is properly cited. The written permission of Cambridge University Press must be obtained for commercial re-use or in order to create a derivative work. 
Several reports have shown that generation may affect chronotype, and elderly people tend to have an earlier chronotype than young people $\mathrm{e}^{(13,14)}$. Therefore, we investigated the association between human chronotype and dietary intake in elderly Japanese people. Furthermore, to clarify the association between chronotype and health-related outcomes in elderly Japanese people, we investigated the correlation between the midpoint of sleep and health-related quality of life (HRQOL) as assessed by the Japanese version short-form 36-item health survey (SF-36) ${ }^{(15-17)}$. The SF-36 has been validated and widely used in the epidemiological studies of HRQoL ${ }^{(18)}$. HRQoL represents a comprehensive perception of physical and mental health. Although a few studies have shown an association between evening chronotype and low HRQoL in adolescents $^{(19)}$ and adults aged 49-79 years ${ }^{(20)}$, the association between HRQoL and chronotype among elderly people has not been investigated. Furthermore, some studies also found an association between chronotype and mental health $^{(21,22)}$. Early chronotypes had a modestly lower risk of depression among middle-aged and elderly US women in the Nurses' Health Study ${ }^{(21)}$. Moreover, the midpoint of sleep on free days was significantly positively correlated with insomnia, depression and anxiety among Korean older adults ${ }^{(2)}$. However, in elderly people, there are no reports investigating the relationship between chronotypes and detailed dietary intake or mental and physical health. Therefore, we investigated the association of chronotype with dietary intake, depression as assessed by the Center Epidemiologic Studies Scale and HRQoL in elderly Japanese women.

\section{Subjects and methods}

\section{Study design}

The data for this study were obtained from the Three-generation Study of Women on Diets and Health. In brief, the design of this study was as follows. The survey was conducted from April to May in 2011 and 2012. A total of 7016 first-year students from eighty-five institutions of higher education in thirty-five of forty-seven prefectures in Japan were asked to answer two questionnaires (dietary habits and other lifestyle factors). The students were also required to distribute these two questionnaires directly to their mothers and grandmothers. If a student subject's grandmother could not participate in the study, a 65-89-year-old female acquaintance was allowed in her place. The collaborators in the Three-generation Study of Women on Diets and Health explained the purpose and outline of the survey. Written informed consent was acquired from all subjects. Other details of the study design have been described in previous reports $^{(23,24)}$.

The subjects of the study were the women of the grandmothers' generation (response rate: $33.2 \%, n$ 2332). Among these subjects' responses, we excluded the data gathered at one institution with a low response rate $(2 \%)$. Because a large earthquake occurred in March 2011 in the northeastern part of Japan, it is suggested that the subjects in this region might not have reported their usual diet and lifestyle during that time. Thus, we excluded subjects who lived in eastern Japan in 2011 ( $n$ 47). Furthermore, we selected only subjects over 65 years old ( $n$ 2260). Other exclusion criteria for the subjects in this study were as follows: energy intake of less than 0.5 times the energy requirement for the lowest physical activity $(<775 \mathrm{kcal} / \mathrm{d}, n 27)$ or more than 1.5 times the energy requirement for the highest physical activity $(>3150 \mathrm{kcal} / \mathrm{d}$, $n$ 44) according to the Dietary Reference Intakes for Japanese, $202{ }^{(25)}$; eating a controlled diet as recommended by a doctor or dietitian ( $n$ 316); a case history of mental health-related diseases ( $n$ 65); use of a psychiatric prescription ( $n$ 59); missing data on place of residence ( $n$ 9) and missing information on height and weight ( $n 5)$, marital status ( $n$ 1), number of people in the household $(n 9)$, alcohol drinking (n 19), physical activity (n 38), Pittsburgh Sleep Quality Index, PSQI ( $n$ 52), Center for Epidemiologic Studies Depression (CES-D) Scale ( $n$ 52), the Japanese version of the SF-36 ( $n$ 26) and sleep-related factors ( $n$ 27), such as the midpoint of sleep falling outside the range of 0:00-12:00 (n 23). After these exclusions, the final sample consisted of 1618 women.

\section{Dietary intake}

We assessed dietary intake over the previous month in terms of energy, food groups, food items and nutrients using a validated instrument, the brief-type self-administered diet history questionnaire (BDHQ) ${ }^{(26,27)}$. The BDHQ has four pages and has fixed portions of fifty-eight food and beverage items. Measures of dietary intake were calculated using an ad hoc computer algorithm for the BDHQ based on the Standard Tables of Food Composition in Japan ${ }^{(28)}$. Additional details on the structure of the BDHQ are described in another report ${ }^{(26,27)}$.

\section{Midpoint of sleep, sleep duration, sleep quality, and depressive symptoms}

Roenneberg et al. reported the midpoint of sleep on the Munich ChronoType Questionnaire as an indicator of chronotype $^{(29,30)}$. In the present study, the midpoint of sleep was calculated as the halfway point between sleep onset time and rise time.

In this study, we used a lifestyle questionnaire booklet that included the PSQI ${ }^{(31)}$, the CES-D scale ${ }^{(32,33)}$, the Japanese version of the SF-36 ${ }^{(15-17)}$ and sleep-related variables for the previous month. The subjects' self-reported bedtime, the time spent in bed until falling asleep and the rise time were included in the lifestyle questionnaire. The sleep onset time was calculated by subtracting the time from bedtime to falling asleep. Sleep duration was also calculated from sleep onset and rise time.

Sleep quality was assessed by the global PSQI score ${ }^{(31)}$. A higher PSQI score indicates poor sleep quality.

Depressive symptoms were assessed by the CES-D ${ }^{(32,33)}$. Subjects with a total CES-D score of $\geq 16$ were defined as having depressive symptoms. 


\section{Health-related quality of life}

The subjects' HRQoL was estimated by the SF-36. The 36-item scale in the SF-36 provides a total of eight subscales: four physical health-related scales (physical functioning (PF); physical role (role-physical: RP); bodily pain (BP); general health perception $(\mathrm{GH})$ ) and four mental health-related scales (vitality (VT); social functioning (SF); role-emotional (RE); mental health perception (MH)). A score from 0 to 100 is calculated for each health scale, where a lower score indicates poorer health status ${ }^{(15-17)}$.

\section{Other variables}

The subjects self-reported other variables on the BDHQ or the lifestyle questionnaire. The subjects reported their birth date, body height and body weight on the BDHQ, and the body mass index (BMI) was calculated as the current body weight $(\mathrm{kg})$ divided by the square of body height $(\mathrm{m})$. The subjects' area of residence was reported in the lifestyle questionnaire. These areas were divided into six regions (Hokkaido and Tohoku; Kanto; Hokuriku and Tokai; Kinki; Chugoku and Shikoku; Kyushu) and into three categories according to population size (city with a population $\geq 1$ million; city with a population $<1$ million; town or village). The subjects also reported whether they lived alone, as well as their marital status (single, married, widowed, or separated), education ( $\leq$ junior high school and others, high school, or $\geq$ college), current smoking status, alcohol consumption, dietary supplement use, prescription use and history of chronic disease. Physical activity was calculated as the average metabolic equivalent (MET) hours, which is based on the self-reported duration of five categorised activities (walking, bicycling, standing, running and high-intensity activities), sitting and sleeping. The MET values were assigned each activity. The MET values of each activity were 3.5 for walking, 7.5 for bicycling, 3.2 for standing, $7 \cdot 0$ for running, $8 \cdot 0$ for high-intensity activities, $1 \cdot 0$ for sleeping and 1.3 for sitting ${ }^{(34)}$.

\section{Statistical analysis}

The subjects in this study were divided into quintiles according to the midpoint of sleep, from the earliest (Q1) to the latest (Q5) quintile. The means of dietary intake, midpoint of sleep, bedtime, rise time, sleep latency, sleep duration, PSQI score, health-related SF-36 score and CES-D score were calculated for each midpoint of sleep quintile. The median values for each midpoint of sleep quintile were tested as continuous variables for linear trends. Macronutrients intake were presented as percentages of total energy intake. Energy-adjusted intake of other nutrients and food groups intake were presented as the value per $1000 \mathrm{kcal}$. The means of dietary intake were adjusted for potential confounding factors. The variables that were significantly correlated with the midpoint of sleep, such as body height, body weight, survey year, residential block, size of residential area, education, physical activity and depression symptoms, were included as confounding factors. We analysed differences between the Q1 group and the other groups using Dunnett's test. A Mantel-Haenszel $\chi^{2}$ test was used to test for differences in proportions for categorical variables.

All data were analysed using the statistical software SAS version 9.4 (SAS Institute Inc., Cary, NC, USA).

\section{Results}

Subject characteristics by midpoint of sleep quintile are shown in Table 1. The mean age (standard deviation, SD) of the subjects was 74.6 (4.9) years, and the mean BMI (SD) was 22.7 (3.1). The group with a later midpoint of sleep was heavier and taller than the group with an earlier midpoint. Survey year, residential block, size of residential area, education and physical activity differed in proportions between groups with different midpoints of sleep. The group with a later midpoint of sleep had higher CES-D scores and indicated more depressive symptoms than the group with an earlier midpoint of sleep.

Sleep status and total PSQI scores are shown in Table 2. The subjects with a later midpoint of sleep had later bedtimes and rise times than those with an earlier midpoint of sleep. Sleep latency was longer and sleep duration was shorter in the group with a later midpoint of sleep than in the group with an earlier midpoint of sleep. The subjects with a later midpoint of sleep had higher PSQI scores.

Total energy intake and specific nutrient and food group intakes are presented in Table 3. A significant negative trend from the earliest (Q1) to the latest (Q5) midpoint of sleep was observed for the intake of vitamin $\mathrm{D}$. A significant positive trend from the earliest (Q1) to the latest (Q5) midpoint of sleep was observed for the intake of bread and caffeinated drinks. Bread and caffeinated drinks significantly increased in the Q5 group compared with the Q1 group. No statistically significant association was observed between energy intake and listed nutrients or other food groups.

The HRQoL results (SF-36 scores) are presented in Table 4. A significant negative trend from the earliest (Q1) to the latest (Q5) midpoint of sleep was observed for the GH score. There were no statistically significant associations between other SF-36 scores and the midpoint of sleep.

\section{Discussion}

There was little association between chronotype and dietary intake in the elderly subjects in this study. The association between the midpoint of sleep and the dietary intake in elderly subjects differed from that in young women in a previous study ${ }^{(11,12)}$. Our two previous studies indicated significant and independent associations. Both a late midpoint of sleep and an evening chronotype as assessed by the MEQ score were associated with an inadequate intake of several vitamins and minerals, a low intake of vegetables or pulses and a high intake of confections. Similarly, another study of a random sample of the population aged 25-74 years in Finland found that individuals with an evening chronotype as assessed by the MEQ score had unfavourable dietary habits ${ }^{(35)}$. Furthermore, in the recent reviews, limited cross-sectional 


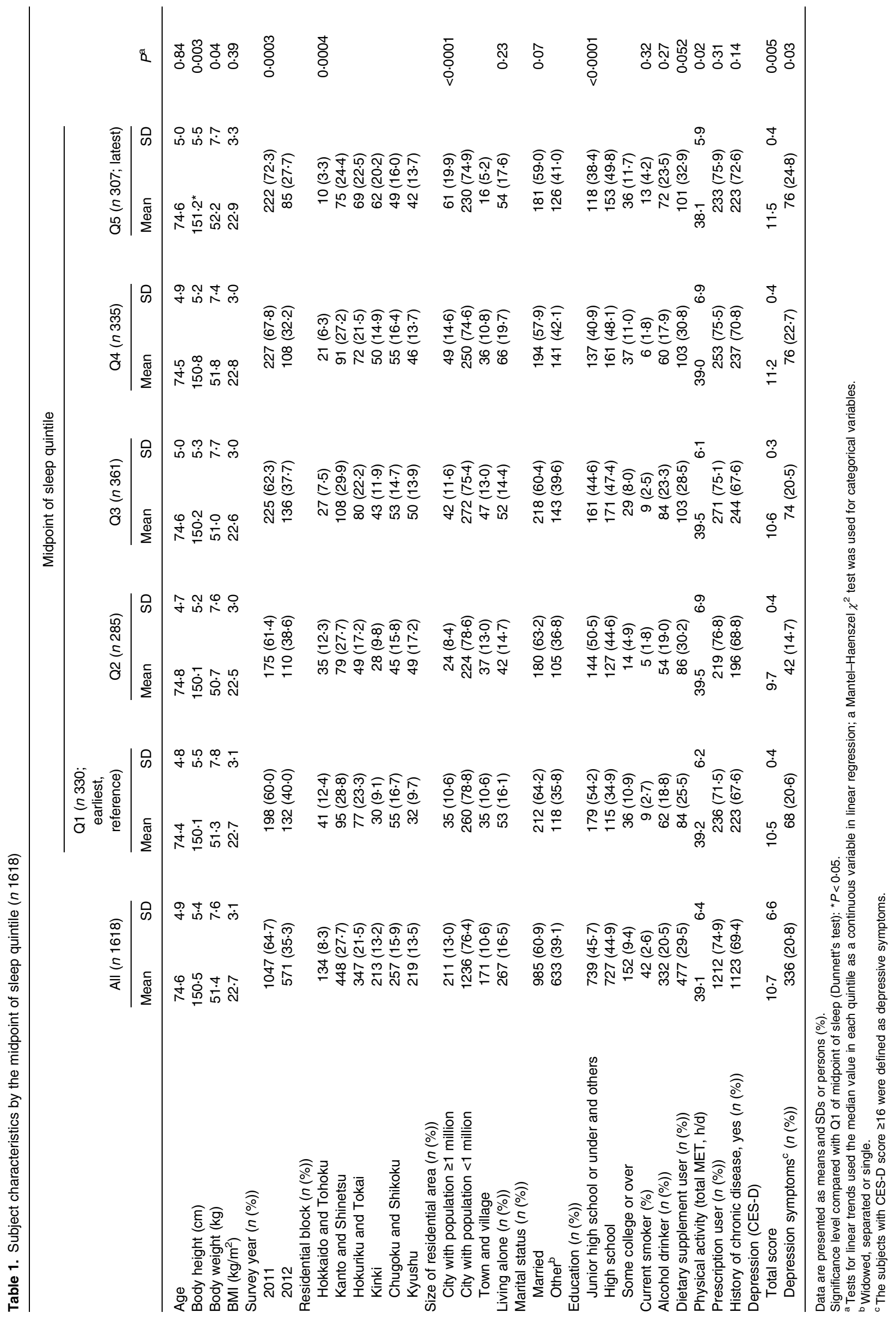


Table 2. Sleep status and total PSQI score by the midpoint of sleep quintile $(n 1618)$

\begin{tabular}{|c|c|c|c|c|c|c|c|c|c|c|c|c|c|}
\hline & & & \multicolumn{10}{|c|}{ Midpoint of sleep quintile } & \multirow{3}{*}{$P^{a}$} \\
\hline & \multicolumn{2}{|c|}{ All $(n$ 1618) } & \multicolumn{2}{|c|}{$\begin{array}{l}\text { Q1 }(n \text { 330; } \\
\text { earliest, } \\
\text { reference) }\end{array}$} & \multicolumn{2}{|c|}{ Q2 (n 285) } & \multicolumn{2}{|c|}{ Q3 (n 361) } & \multicolumn{2}{|c|}{ Q4 (n 335) } & \multicolumn{2}{|c|}{$\begin{array}{c}\text { Q5 ( } n \text { 307; } \\
\quad \text { latest) }\end{array}$} & \\
\hline & Mean & SD & Mean & SD & Mean & SD & Mean & SD & Mean & SD & Mean & SD & \\
\hline Midpoint of sleep (o'clock; h:min) & $2: 28$ & $0: 52$ & $1: 19$ & $0: 23$ & $2: 00^{\star *}$ & $0: 07$ & $2: 26$ ** & $0: 08$ & $2: 53^{\star \star}$ & $0: 08$ & $3: 41^{\star *}$ & $0: 34$ & $<0.0001$ \\
\hline Bedtime (o'clock; h:min) & $22: 16$ & 1:05 & $21: 04$ & $0: 43$ & $21: 49^{* *}$ & $0: 43$ & $22: 20$ ** & $0: 39$ & $22: 44^{\star *}$ & $0: 40$ & $23: 25^{\star *}$ & $0: 55$ & $<0.0001$ \\
\hline Rise time (o'clock; h:min) & $6: 13$ & $0: 57$ & $5: 12$ & $0: 42$ & $5: 52^{\star *}$ & $0: 36$ & $6: 08^{* *}$ & $0: 32$ & $6: 36^{\star *}$ & $0: 35$ & $7: 19^{\star \star}$ & $0: 46$ & $<0.0001$ \\
\hline Sleep latency (hours; h.min) & 0.25 & 0.29 & 0.22 & 0.22 & 0.19 & 0.20 & 0.22 & 0.20 & 0.26 & 0.22 & $0.40^{\star *}$ & 0.49 & $<0.0001$ \\
\hline Sleep duration ${ }^{\mathrm{b}}$ (hours; h.min) & $7 \cdot 31$ & $1 \cdot 10$ & $7 \cdot 47$ & 1.07 & $7 \cdot 44$ & $1 \cdot 13$ & $7 \cdot 25^{\star \star}$ & 1.04 & $7 \cdot 26^{* *}$ & 1.08 & $7 \cdot 15^{\star \star}$ & 1.15 & $<0.0001$ \\
\hline Total PSQI score & $5 \cdot 3$ & $3 \cdot 1$ & 4.8 & $2 \cdot 8$ & 4.6 & $2 \cdot 8$ & $5 \cdot 1$ & 3.0 & $5 \cdot 6^{*}$ & $3 \cdot 1$ & $6 \cdot 5^{\star *}$ & 3.4 & $<0.0001$ \\
\hline
\end{tabular}

PSQI, Pittsburgh Sleep Quality Index.

Data are presented as means and SDs.

Significance level compared with Q1 of the midpoint of sleep (Dunnett's test): ${ }^{\star} P<0.01$; ${ }^{\star \star} P<0.001$.

${ }^{a}$ Tests for linear trends used the median value in each quintile as a continuous variable in linear regression.

${ }^{\mathrm{b}}$ It is calculated with rise time and bedtime adjusted for sleep latency.

studies suggest that an evening chronotype is associated with a low intake of vegetables and fruit and a high intake of several beverages, e.g., energy, alcoholic, sugary and caffeinated drinks, in adolescents and adults ${ }^{(9,10)}$. However, there are few such studies in young children and older adults. The elderly subjects in this study with a late midpoint of sleep did not have the same characteristics of dietary intake as the young subjects in our previous study ${ }^{(11,12)}$. A late midpoint of sleep was associated with the intake of only a few nutrient and food groups: lower intake of vitamin D and higher intake of bread and caffeinated drinks (including green tea, black tea, oolong tea, coffee and cola). In young women in our previous study, a long sleep duration was associated with a late midpoint of sleep ${ }^{(11)}$. In contrast, a short sleep duration was associated with a late midpoint of sleep in the elderly women in this study. Reviews by cross-sectional studies revealed that a short sleep duration was associated with inadequate dietary habits in adults and children ${ }^{(36,37)}$. On the other hand, one Japanese study showed that sleep duration was associated with dietary nutrient intake in middle-aged men but not in women ${ }^{(38)}$. A recent report also showed that the relationship between diet and sleep duration differed between pre-/ perimenopausal and postmenopausal women ${ }^{(39)}$. In this study, the difference in sleep duration among chronotypes was less than $1 \mathrm{~h}$, and the mean sleep duration in all groups was above $7 \mathrm{~h}$. The National Sleep Foundation of the USA reported that an adequate sleep duration is $7-8 \mathrm{~h}$ and that a sleep duration of less than $5 \mathrm{~h}$ is insufficient for optimal health in older adults ${ }^{(40)}$. Therefore, although the subjects with a late midpoint of sleep had short sleep duration, it might be sufficient for them. On the other hand, the subjects with a late midpoint of sleep also had poor sleep quality in this study. A previous report of the Three-generation Study of Women on Diets and Health Study Group showed that poor sleep quality was associated with low intake of vegetables, high intake of confectionary and unhealthy eating habits among middle-aged female Japanese workers ${ }^{(41)}$. Further research is needed to clarify the relationship among generational and sexspecific sleep characteristics, chronotypes and dietary intake.
Weak correlations were observed between HRQoL indicators and chronotype in this study. One of the physical health components of the SF-36, GH, was poor in subjects with a late midpoint of sleep compared with subjects with an early midpoint of sleep. No significant associations were observed between other physical and mental health components and the midpoint of sleep. Although GH includes physical health components, it has been reported that this scale is valid for assessing the combined effects of physical and mental conditions in Japanese people ${ }^{(15,16)}$. A recent study reported that young adults' sleep quality is more closely associated with mental and physical health than their chronotype or sleep duration $^{(42)}$. It was also reported that lower self-reported sleep quantity and quality were associated with poor HRQoL in children, but device-measured sleep characteristics were not ${ }^{(43)}$. However, although elderly subjects with a late midpoint of sleep had higher sleep scores, indicating poor sleep quality, a late midpoint of sleep was associated only with low GH on the SF-36 in the present study. On the other hand, the ratio of depressive symptoms assessed by the CES-D and the total CES-D score was higher in the group with a late midpoint in the present study. Consistent with the present results, several reports have shown that an evening chronotype is associated with depression ${ }^{(44,45)}$. Several reports also suggest a protective effect of coffee or caffeine drinks against the risk of depression $^{(46,47)}$. A previous report of the Three-generation Study of Women on Diets and Health Study Group also showed an inverse association between depressive symptoms and coffee intake, but not another caffeinated drink, green tea, in elderly Japanese women ${ }^{(48)}$. As mentioned earlier, an association between a higher intake of caffeinated drinks and a late midpoint of sleep was observed in our present study, consistent with other studies ${ }^{(20,49)}$. In the present study, as caffeinated drink intake categorised by the midpoint of sleep was adjusted by depressive symptoms, a higher intake of caffeine may be characteristic of a late midpoint of sleep. Another systematic review reported that poor sleep quality and short sleep duration were associated with high coffee and caffeinated drink intake, and the sleep of older adults may be more 
Table 3. Total energy, nutrients and food group intakes by the midpoint of sleep quintile $(n 1618)$

\begin{tabular}{|c|c|c|c|c|c|c|c|c|c|c|c|c|c|}
\hline & & & \multicolumn{10}{|c|}{ Midpoint of sleep quintile } & \multirow[b]{3}{*}{$P^{a}$} \\
\hline & \multicolumn{2}{|c|}{ All $(n$ 1618) } & \multicolumn{2}{|c|}{$\begin{array}{l}\text { Q1 ( } n \text { 330; } \\
\text { earliest, } \\
\text { reference) }\end{array}$} & \multicolumn{2}{|c|}{ Q2 (n 285) } & \multicolumn{2}{|c|}{ Q3 (n 361) } & \multicolumn{2}{|c|}{ Q4 (n 335) } & \multicolumn{2}{|c|}{$\begin{array}{l}\text { Q5 ( } n \text { 307; } \\
\quad \text { latest) }\end{array}$} & \\
\hline & Mean & SD & Mean & SE & Mean & SE & Mean & SE & Mean & SE & Mean & SE & \\
\hline Energy (kcal/d) & 1737 & 457 & 1720 & 25 & 1782 & 27 & 1736 & 24 & 1745 & 24 & 1700 & 26 & 0.4203 \\
\hline \multicolumn{14}{|l|}{ Nutrients } \\
\hline Protein (\% energy) & $16 \cdot 9$ & 3.2 & $16 \cdot 8$ & 0.2 & $17 \cdot 3$ & 0.2 & $16 \cdot 9$ & 0.2 & $16 \cdot 6$ & 0.2 & $16 \cdot 7$ & 0.2 & 0.1194 \\
\hline Fat (\% energy) & $25 \cdot 6$ & $5 \cdot 2$ & $25 \cdot 4$ & 0.3 & $25 \cdot 9$ & 0.3 & $25 \cdot 3$ & 0.3 & $25 \cdot 4$ & 0.3 & $26 \cdot 0$ & 0.3 & 0.3195 \\
\hline Carbohydrates (\% energy) & $56 \cdot 0$ & $7 \cdot 4$ & $56 \cdot 2$ & 0.4 & $55 \cdot 1$ & 0.4 & $56 \cdot 3$ & 0.4 & $56 \cdot 4$ & 0.4 & $55 \cdot 7$ & 0.4 & 0.9057 \\
\hline Cholesterol (mg/1000 kcal) & 230 & 76 & 229 & 4 & 236 & 5 & 227 & 4 & 229 & 4 & 230 & 4 & 0.7830 \\
\hline Sodium (mg/1000 kcal) & 2564 & 508 & 2606 & 28 & 2588 & 30 & 2566 & 27 & 2517 & 28 & 2549 & 29 & 0.0525 \\
\hline Potassium (mg/1000 kcal) & 1625 & 393 & 1617 & 21 & 1646 & 23 & 1637 & 20 & 1613 & 21 & 1612 & 22 & 0.6173 \\
\hline Calcium (mg/1000 kcal) & 366 & 113 & 367 & 6 & 375 & 7 & 370 & 6 & 360 & 6 & 359 & 6 & 0.1948 \\
\hline Magnesium (mg/1000 kcal) & 159 & 33 & 159 & 2 & 162 & 2 & 160 & 2 & 157 & 2 & 157 & 2 & 0.1443 \\
\hline Iron (mg/1000 kcal) & $5 \cdot 1$ & $1 \cdot 1$ & $5 \cdot 1$ & 0.1 & $5 \cdot 2$ & 0.1 & $5 \cdot 1$ & 0.1 & $5 \cdot 1$ & 0.1 & $5 \cdot 1$ & 0.1 & 0.6400 \\
\hline Zinc (mg/1000 kcal) & $4 \cdot 8$ & 0.6 & $4 \cdot 8$ & 0.0 & 4.9 & 0.0 & $4 \cdot 8$ & 0.0 & $4 \cdot 8$ & 0.0 & $4 \cdot 7$ & 0.0 & 0.1307 \\
\hline Retinol equivalent $(\mu \mathrm{g} / 1000 \mathrm{kcal})$ & 474 & 260 & 457 & 14 & 502 & 15 & 466 & 14 & 470 & 14 & 479 & 15 & 0.6297 \\
\hline Vitamin D ( $\mu \mathrm{g} / 1000 \mathrm{kcal})$ & $11 \cdot 1$ & $6 \cdot 2$ & $11 \cdot 3$ & 0.30 & $11 \cdot 8$ & 0.4 & $11 \cdot 1$ & 0.3 & $10 \cdot 7$ & 0.3 & $10 \cdot 7$ & 0.4 & 0.0498 \\
\hline Vitamin E (mg/1000 kcal) & 4.4 & 1.0 & 4.4 & 0.1 & $4 \cdot 4$ & 0.1 & 4.4 & 0.1 & 4.4 & 0.1 & 4.5 & 0.1 & 0.3455 \\
\hline Thiamin $(\mathrm{mg} / 1000 \mathrm{kcal})$ & 0.46 & 0.09 & 0.46 & 0.00 & 0.47 & 0.01 & 0.47 & 0.00 & 0.46 & 0.00 & 0.46 & 0.01 & 0.9215 \\
\hline Riboflavin (mg/1000 kcal) & 0.81 & 0.19 & 0.79 & 0.01 & $0.83^{*}$ & 0.10 & 0.81 & 0.01 & 0.81 & 0.01 & 0.81 & 0.01 & 0.5611 \\
\hline Vitamin $B_{6}(\mathrm{mg} / 1000 \mathrm{kcal})$ & 0.8 & 0.2 & 0.8 & 0.0 & 0.8 & 0.0 & 0.8 & 0.0 & 0.8 & 0.0 & 0.8 & 0.0 & 0.3682 \\
\hline Vitamin $B_{12}(\mu \mathrm{g} / 1000 \mathrm{kcal})$ & $6 \cdot 6$ & 3.0 & 6.5 & 0.2 & $6 \cdot 9$ & 0.2 & 6.5 & 0.2 & 6.5 & 0.2 & $6 \cdot 4$ & 0.2 & 0.3978 \\
\hline Folate $(\mu \mathrm{g} / 1000 \mathrm{kcal})$ & 238 & 72 & 234 & 4 & 241 & 4.0 & 240 & 4 & 238 & 4 & 239 & 4 & 0.5034 \\
\hline Vitamin C (mg/1000 kcal) & 85 & 30 & 83 & 2 & 84 & 2 & 86 & 2 & 86 & 2 & 85 & 2 & 0.1555 \\
\hline \multicolumn{14}{|l|}{ Food groups ( $\mathrm{g} / 1000 \mathrm{kcal})$} \\
\hline Rice & 167.4 & $69 \cdot 7$ & $167 \cdot 6$ & 3.7 & $166 \cdot 7$ & 4.0 & $172 \cdot 8$ & 3.5 & $170 \cdot 4$ & $3 \cdot 6$ & $158 \cdot 4$ & 3.9 & 0.2210 \\
\hline Noodles ${ }^{b}$ & $30 \cdot 1$ & 23.4 & $30 \cdot 1$ & 1.3 & $28 \cdot 5$ & 1.4 & 29.5 & $1 \cdot 2$ & $29 \cdot 1$ & $1 \cdot 3$ & 33.5 & $1 \cdot 3$ & 0.0886 \\
\hline Bread & $21 \cdot 2$ & $16 \cdot 0$ & 20.9 & 0.9 & $18 \cdot 1$ & 0.9 & $20 \cdot 4$ & 0.8 & $21 \cdot 0$ & 0.9 & $25 \cdot 3^{*}$ & 0.9 & 0.0001 \\
\hline Confections $^{c}$ & 32.9 & $22 \cdot 4$ & $34 \cdot 0$ & $1 \cdot 2$ & $32 \cdot 8$ & $1 \cdot 3$ & 31.5 & $1 \cdot 2$ & 34.0 & $1 \cdot 2$ & $32 \cdot 1$ & $1 \cdot 3$ & 0.4181 \\
\hline Potatoes & 32.9 & 23.7 & 33.8 & $1 \cdot 3$ & $34 \cdot 8$ & $1 \cdot 4$ & 33.0 & $1 \cdot 2$ & $32 \cdot 2$ & $1 \cdot 3$ & $31 \cdot 0$ & 1.4 & 0.0686 \\
\hline Fruits $^{d}$ & $78 \cdot 2$ & $56 \cdot 0$ & 75.9 & 3.0 & 72.5 & 3.3 & $80 \cdot 6$ & $2 \cdot 9$ & $79 \cdot 7$ & $3 \cdot 0$ & 81.5 & $3 \cdot 2$ & 0.0722 \\
\hline Vegetables $^{\mathrm{e}}$ & $184 \cdot 3$ & 80.0 & $182 \cdot 7$ & $4 \cdot 3$ & $186 \cdot 3$ & $4 \cdot 7$ & 185.9 & $4 \cdot 1$ & $184 \cdot 7$ & $4 \cdot 3$ & $181 \cdot 8$ & $4 \cdot 6$ & 0.8645 \\
\hline Soya & $41 \cdot 6$ & $25 \cdot 1$ & $41 \cdot 8$ & 1.4 & $44 \cdot 9$ & 1.5 & $41 \cdot 1$ & $1 \cdot 3$ & $41 \cdot 0$ & 1.4 & $39 \cdot 4$ & 1.4 & 0.0765 \\
\hline Fish and shellfish ${ }^{f}$ & 58.9 & $30 \cdot 7$ & $59 \cdot 6$ & 1.7 & $61 \cdot 7$ & 1.8 & $58 \cdot 6$ & $1 \cdot 6$ & $57 \cdot 6$ & 1.7 & $57 \cdot 2$ & 1.8 & 0.1369 \\
\hline Meat $^{9}$ & $32 \cdot 0$ & $17 \cdot 8$ & 30.7 & $1 \cdot 0$ & $34 \cdot 1$ & $1 \cdot 1$ & $32 \cdot 2$ & 0.9 & $30 \cdot 8$ & $1 \cdot 0$ & $32 \cdot 6$ & $1 \cdot 0$ & 0.6915 \\
\hline Eggs & $20 \cdot 1$ & $12 \cdot 6$ & 19.5 & 0.7 & 20.5 & 0.8 & $19 \cdot 4$ & 0.7 & $20 \cdot 7$ & 0.7 & $20 \cdot 4$ & 0.7 & 0.4080 \\
\hline Milk and milk products & $73 \cdot 1$ & $56 \cdot 4$ & $71 \cdot 7$ & $3 \cdot 1$ & $74 \cdot 2$ & 3.4 & 74.7 & 3.0 & $72 \cdot 3$ & $3 \cdot 1$ & $72 \cdot 4$ & $3 \cdot 3$ & 0.9701 \\
\hline Caffeine drinks $^{\mathrm{h}}$ & $347 \cdot 1$ & $172 \cdot 7$ & 322.5 & 9.5 & $337 \cdot 2$ & $10 \cdot 2$ & $350 \cdot 2$ & $9 \cdot 0$ & $349 \cdot 4$ & $9 \cdot 4$ & $376 \cdot 5^{\star \star}$ & 9.9 & $<0.0001$ \\
\hline
\end{tabular}

'All' data are means and SDs. Q1-Q5 data are means and SEs. Data of Q1-Q5 were adjusted for body height, body weight, survey year (2011 or 2012), residential block (Hokkaido and Tohoku, Kanto, Hokuriku and Tokai, Kinki, Chugoku and Shikoku, and Kyushu), size of residential area (city with population $\geq 1$ million, city with population $<1$ million, or town and village), education (junior high school or under and others, high school or some college or over), physical activity (total MET, h/d) and depression symptoms (yes or no). Significance level compared with Q1 of midpoint of sleep (Dunnett's test): ${ }^{\star} P<0.01 ;{ }^{\star \star} P<0.001$.

${ }^{a}$ Tests for linear trend used the median value in each quintile as a continuous variable in linear regression.

${ }^{\mathrm{b}}$ Including Japanese noodles (buckwheat and Japanese wheat noodles), instant noodles, Chinese noodles and pasta.

${ }^{\mathrm{c}}$ Including Western-style cake, cookies, Japanese-style cake, rice crackers, rice cakes, Japanese-style pancakes and ice cream.

${ }^{\mathrm{d}}$ Including citrus fruits, persimmon, strawberry, kiwi fruit and other all fruits.

e Including coloured vegetables, other vegetables, mushrooms and sea vegetables.

${ }^{\mathrm{f}}$ Including cuttlefish, octopus, lobster, shellfish, small fish, canned tuna, dried fish, salt fish, oily fish and non-oily fish.

${ }^{g}$ Including chicken, pork, beef, ham, sausage, bacon and liver.

${ }^{\mathrm{h}}$ Including green tea, black tea, oolong tea, coffee and cola.

affected than the sleep of younger adults ${ }^{(50)}$. Our elderly subjects with a late midpoint of sleep had both poor sleep quality and a short sleep duration compared with subjects with an early midpoint of sleep. Further research is needed to determine its protective effect on HRQoL except GH and the interaction of chronotype properties such as sleep quality and sleep duration.

Fukuhara and Suzukamo reported Japanese population norms during a validation of the Japanese version of the SF-36 ${ }^{(17)}$. Although a late midpoint was associated with low $\mathrm{GH}$ score in this study, the means of all eight SF-36 subscale scores for elderly women older than 65 years in this study exceeded the norms for the Japanese female population aged 70-79 years. These results revealed that HRQoL might be maintained in the subjects in this study. It has been reported that patients with several chronic diseases have poor HRQOL as assessed by the SF-36 $6^{(51,52)}$. A recent randomised controlled trial showed that a 6 -month reduced-calorie diet improved the HRQoL score in older adults with obesity ${ }^{(53)}$. A previous report of the Three-generation Study of Women on Diets and Health Study Group showed that frailty assessed using the PF and VT scales of the SF-36 was inversely associated with the intake of protein, green tea, coffee, vegetables and fruits ${ }^{(23)}$. Furthermore, short or long sleep duration was 
Table 4. HRQoL results by the midpoint of sleep quintile ( $n$ 1618)

\begin{tabular}{|c|c|c|c|c|c|c|c|c|c|c|c|c|c|}
\hline & \multicolumn{2}{|c|}{ SF-36 subscale } & \multicolumn{10}{|c|}{ Midpoint of sleep quintile } & \multirow[b]{3}{*}{$P^{a}$} \\
\hline & \multicolumn{2}{|c|}{ All $(n$ 1618) } & \multicolumn{2}{|c|}{$\begin{array}{c}\text { Q1 ( } n \text { 330; } \\
\text { earliest, reference) }\end{array}$} & \multicolumn{2}{|c|}{ Q2 (n 285) } & \multicolumn{2}{|c|}{ Q3 (n 361) } & \multicolumn{2}{|c|}{ Q4 (n 335) } & \multicolumn{2}{|c|}{$\begin{array}{l}\text { Q5 }(n 307 ; \\
\quad \text { latest) }\end{array}$} & \\
\hline & Mean & SD & Mean & SD & Mean & SD & Mean & SD & Mean & SD & Mean & SD & \\
\hline PF & $76 \cdot 1$ & 21.3 & $75 \cdot 9$ & 22.3 & $77 \cdot 8$ & 21.1 & 77.5 & 19.3 & $75 \cdot 2$ & 21.6 & 73.8 & 22.0 & 0.11 \\
\hline $\mathrm{RP}$ & $83 \cdot 0$ & 22.9 & $83 \cdot 7$ & 22.5 & 83.8 & 23.4 & $83 \cdot 6$ & 21.3 & $82 \cdot 7$ & 24.3 & $81 \cdot 2$ & 23.3 & 0.15 \\
\hline BP & 68.5 & 22.3 & $68 \cdot 8$ & 22.3 & $69 \cdot 3$ & 21.9 & 67.9 & 21.2 & $68 \cdot 1$ & 22.5 & $68 \cdot 3$ & 23.9 & 0.62 \\
\hline $\mathrm{GH}$ & 63.6 & 19.1 & $64 \cdot 9$ & 19.4 & $65 \cdot 8$ & 18.9 & $64 \cdot 2$ & 18.2 & $61 \cdot 8$ & 19.3 & 61.4 & 19.3 & 0.002 \\
\hline VT & $67 \cdot 1$ & 19.9 & $67 \cdot 7$ & 21.3 & $68 \cdot 0$ & 19.9 & $68 \cdot 1$ & 19.0 & 65.5 & 19.6 & $66 \cdot 1$ & 19.6 & 0.13 \\
\hline SF & $88 \cdot 6$ & 17.7 & 87.5 & 19.3 & 88.7 & 19.1 & 88.9 & 16.9 & $88 \cdot 8$ & 16.9 & 89.0 & 16.7 & 0.30 \\
\hline RE & $86 \cdot 8$ & 21.1 & $86 \cdot 6$ & 22.0 & 89.0 & 19.8 & 87.2 & 20.6 & $86 \cdot 1$ & 22.5 & $85 \cdot 1$ & 20.5 & 0.15 \\
\hline $\mathrm{MH}$ & 76.9 & 18.0 & 77.1 & 18.4 & 77.9 & 18.4 & $77 \cdot 3$ & 17.4 & $76 \cdot 0$ & 18.3 & $76 \cdot 0$ & 17.7 & 0.23 \\
\hline
\end{tabular}

HRQoL, health-related quality of life.

PF, physical functioning. RP, role-physical. BP, bodily pain. GH, general health perception. VT, vitality. SF, social functioning. RE, role-emotional. MH, mental health perception. Data are means and SDs.

${ }^{a}$ Tests for linear trend used the median value in each quintile as a continuous variable in linear regression.

independently associated with impairment of HRQoL in adults with chronic diseases ${ }^{(54,55)}$. In the present study, although more than $60 \%$ of the subjects had a history of chronic disease, the details of their diseases were not investigated. Further studies are needed to confirm the association among chronotype, sleep characteristics (including sleep duration and sleep quality), HRQoL and dietary intake in elderly people with specific chronic diseases.

The present study had several limitations. First, we did not ask the subjects about their sleep habits on workdays and free days separately. We calculated the midpoint of sleep using bedtime and rise time on most days of the previous month. We also did not ask whether the subjects had an occupation. The midpoint of sleep differs between free days and workdays, and the MEQ score on the Horne-Ostberg questionnaire appears to correlate better with the midpoint of sleep on free days than on workdays ${ }^{(29,30)}$. Although it may be suggested that the difference between free days and workdays was small because the subjects were elderly women aged above 65 years, it is possible that their occupation, or any other regular schedule, affected their chronotype as assessed by the midpoint of sleep. Second, we did not investigate seasonable variables in the results. It was reported that chronotype, depression and dietary intake were affected by season $^{(56-58)}$. Because our data were collected in spring, the seasonal variability of these parameters may affect the results in the present study. Third, it is possible that the elderly women in the present study may not sufficiently reflect the general population of elderly women in Japan, because most subjects had grandchildren who were students at universities, colleges or technical schools. Fourth, the subjects in the present study were elderly women. Although a recent report suggests that the difference in chronotype between men and women diminishes with age, the results for men require further confirmation ${ }^{(59)}$. Finally, the present findings were observed by a cross-sectional study. Longitudinal studies are needed to confirm that chronotype directly affects dietary intake, depression and HRQoL in elderly people.

In conclusion, a late midpoint of sleep was associated with a lower intake of vitamin D and a higher intake of bread and caffeinated drinks. No correlations were observed between chronotype and other nutrient or food intakes. Subjects with a late midpoint of sleep showed low GH and a high CES-D score. Other HRQoL scores were not different between the groups with different midpoints. In this study, the subjects with a late midpoint of sleep had poor sleep quality and a shorter sleep duration than those with an early midpoint of sleep. These results suggest that it is necessary to consider the relationship between chronotype and diet and health, taking into consideration sleep characteristics by age.

\section{Acknowledgments}

In this study, we used computing services of the Academic Center for Computing and Media Studies (ACCMS), Kyoto University, for statistical analysis. We thank the members of the Three-generation Study of Women on Diets and Health Study Group as co-authors. All members are listed in the previous report ${ }^{(23)}$.

This study was supported by JSPS KAKENHI under Grant No. 22240077 (to S.S.) from the Japan Society for the Promotion of Science. The data analysis in the present study was also supported in part by JSPS KAKENHI under Grant No. 26350130 (to N.M.) from the Japan Society for the Promotion of Science. The Japan Society for the Promotion of Science had no role in the design of the study, the analysis of the results or the writing of the manuscript.

N.M. collected the baseline data, analysed the data, designed this study and wrote the manuscript. E.F. collected the baseline data and edited and reviewed the manuscript. S.S. contributed to the data collection and management, designed the Three-generation Study of Women on Diets and Health Study Group, and edited and reviewed the manuscript.

This study was conducted according to the guidelines stated in the Declaration of Helsinki, and all procedures for the Three-generation Study of Women on Diets and Health were approved by the Ethics Committee of the University of Tokyo Faculty and Medicine (No. 3249). Written informed consent was obtained from all subjects.

All authors declare that they have no conflicts of interest. 


\section{References}

1. Bray MS \& Young ME (2007) Circadian rhythms in the development of obesity: potential role for the circadian clock within the adipocyte. Obes Rev 8, 169-181.

2. Maury E, Ramsey KM \& Bass J (2010) Circadian rhythms and metabolic syndrome: from experimental genetics to human disease. Circ Res 106, 447-462.

3. Sahar S \& Sassone-Corsi P (2009) Metabolism and cancer: the circadian clock connection. Nat Rev Cancer 9, 886-896.

4. Thomas C \& Power C (2010) Shift work and risk factors for cardiovascular disease: a study at age 45 years in the 1958 British birth cohort. Eur J Epidemiol 25, 305-314.

5. McNeil J, Doucet E \& Chaput JP (2013) Inadequate sleep as a contributor to obesity and type 2 diabetes. Can J Diabetes 37, 103-108.

6. Liu Y, Wheaton AG, Chapman DP, et al. (2013) Sleep duration and chronic diseases among U.S. adults age 45 years and older: evidence from the 2010 behavioral risk factor surveillance system. Sleep $\mathbf{3 6}$, 1421-1427.

7. Fatima Y, Doi SA \& Mamun AA (2015) Longitudinal impact of sleep on overweight and obesity in children and adolescents: a systematic review and bias-adjusted meta-analysis. Obes Rev 16, 137-149.

8. Knutson KL \& von Schantz M (2018) Associations between chronotype, morbidity and mortality in the UK Biobank cohort. Chronobiol Int 35, 1045-1053.

9. Mazri FH, Manaf ZA, Shahar S, et al. (2019) The association between chronotype and dietary pattern among adults: a scoping review. Int J Environ Res Public Health 17, E68.

10. Almoosawi S, Vingeliene S, Gachon F, et al. (2019) Chronotype: implications for epidemiologic studies on chrono-nutrition and cardiometabolic health. Adv Nutr 10, 30-42.

11. Sato-Mito N, Sasaki S, Murakami K, et al. (2011) The midpoint of sleep is associated with dietary intake and dietary behavior among young Japanese women. Sleep Med 12, 289-294.

12. Sato-Mito N, Shibata S, Sasaki S, et al. (2011) Dietary intake is associated with human chronotype as assessed by both morningness-eveningness score and preferred midpoint of sleep in young Japanese women. Int J Food Sci Nutr 62, 525-532.

13. Fischer D, Lombardi DA, Marucci-Wellman H, et al. (2017) Chronotypes in the US - influence of age and sex. PLaS One 12, e0178782.

14. Schuster M, Oberlinner C \& Claus M (2019) Shift-specific associations between age, chronotype and sleep duration. Chronobiol Int 36, 784-795.

15. Fukuhara S, Bito S, Green J, et al. (1998) Translation, adaptation, and validation of the SF-36 health survey for use in Japan. J Clin Epidemiol 51, 1037-1044.

16. Fukuhara S, Ware JE Jr, Kosinski M, et al. (1998) Psychometric and clinical tests of validity of the Japanese SF-36 health survey. J Clin Epidemiol 51, 1045-1053.

17. Fukuhara S \& Suzukamo Y (2004) Manual of SF-36v2 Japanese Version. Kyoto: Institute for Health Outcomes \& Process Evaluation Research (in Japanese).

18. Busija L, Pausenberger E, Haines TP, et al. (2011) Adult measures of general health and health-related quality of life: Medical Outcomes Study Short Form 36-Item (SF-36) and Short Form 12-Item (SF-12) Health Surveys, Nottingham Health Profile (NHP), Sickness Impact Profile (SIP), Medical Outcomes Study Short Form 6D (SF-6D), Health Utilities Index Mark 3 (HUI3), Quality of Well-Being Scale (QWB), and Assessment of Quality of Life (AQol). Arthritis Care Res 63, S383-S412.

19. Roeser K, Bruckner D, Schwerdtle B, et al. (2012) Health-related quality of life in adolescent chronotypes - a model for the effects of sleep problems, sleep-related cognitions, and self-efficacy. Chronobiol Int 29, 1358-1365.

20. Suh S, Yang HC, Kim N, et al. (2017) Chronotype differences in health behaviors and health-related quality of life: a populationbased study among aged and older adults. Behav Sleep Med 15, 361-376.
21. Vetter C, Chang SC, Devore EE, et al. (2018) Prospective study of chronotype and incident depression among middle- and older-aged women in the nurses' health study II. J Psychiatr Res 103, 156-160.

22. Ryu H, Joo EY, Choi SJ, et al. (2018) Validation of the Munich ChronoType Questionnaire in Korean older adults. Psycbiatry Investig 15, 775-782.

23. Kobayashi S, Asakura K, Suga H, et al. (2014) Inverse association between dietary habits with high total antioxidant capacity and prevalence of frailty among elderly Japanese women: a multicenter cross-sectional study. J Nutr Health Aging 18, 827-839.

24. Kobayashi S, Asakura K, Suga H, et al. (2013) High protein intake is associated with low prevalence of frailty among old Japanese women: a multicenter cross-sectional study. Nutr J 12, 164.

25. Ministry of Health, Labour, and Welfare (2019) Dietary Reference Intakes for Japanese, 2020. Tokyo: Ministry of Health, Labour, and Welfare (in Japanese).

26. Kobayashi S, Murakami K, Sasaki S, et al. (2011) Comparison of relative validity of food group intakes estimated by comprehensive and brief-type self-administered diet history questionnaires against 16 d dietary records in Japanese adults. Public Health Nutr 14, $1200-1211$

27. Kobayashi S, Honda S, Murakami K, et al. (2012) Both comprehensive and brief self-administered diet history questionnaires satisfactorily rank nutrient intakes in Japanese adults. J Epidemiol 22, 151-159.

28. Science and Technology Agency (2010) Standard Tables of Food Composition in Japan. Tokyo: Ministry of Education, Culture, Sports, Science and Technology (in Japanese).

29. Zavada A, Gordijn MC, Beersma DG, et al. (2005) Comparison of the Munich Chronotype Questionnaire with the Horne-Ostberg's Morningness-Eveningness Score. Chronobiol Int 22, 267-278.

30. Roenneberg T, Kuehnle T, Juda M, et al. (2007) Epidemiology of the human circadian clock. Sleep Med Rev 11, 429-438.

31. Buysse DJ, Reynolds CF 3rd, Monk TH, et al. (1989) The Pittsburgh Sleep Quality Index: a new instrument for psychiatric practice and research. Psychiatry Res 28, 193-213.

32. Wada K, Tanaka K, Theriault G, et al. (2007) Validity of the Center for Epidemiologic Studies Depression Scale as a screening instrument of major depressive disorder among Japanese workers. $A m$ J Ind Med 50, 8-12.

33. Radloff LS (1977) The CES-D scale: a self-report depression scale for research in the general population. Appl Psychol Meas 1, 385-401.

34. Ainsworth BE, Haskell WL, Herrmann SD, et al. (2011) 2011 Compendium of physical activities: a second update of codes and MET values. Med Sci Sports Exerc 43, 1575-1581.

35. Kanerva N, Kronholm E, Partonen T, et al. (2012) Tendency toward eveningness is associated with unhealthy dietary habits. Chronobiol Int 29, 920-927.

36. Dashti HS, Scheer FA, Jacques PF, et al. (2015) Short sleep duration and dietary intake: epidemiologic evidence, mechanisms, and health implications. Adv Nutr 6, 648-659.

37. Cordova FV, Barja S \& Brockmann PE (2018) Consequences of short sleep duration on the dietary intake in children: a systematic review and metanalysis. Sleep Med Rev 42, 68-84.

38. Komada Y, Narisawa H, Ueda F, et al. (2017) Relationship between self-reported dietary nutrient intake and self-reported sleep duration among Japanese adults. Nutrients 9, E134.

39. Zhu B, Grandner MA, Jackson NJ, et al. (2021) Associations between diet and sleep duration in different menopausal stages. West J Nurs Res, doi: 10.1177/193945920986788 (Online ahead of print).

40. Hirshkowitz M, Whiton K, Albert SM, et al. (2015) National sleep foundation's sleep time duration recommendations: methodology and results summary. Sleep Health 1, 40-43.

41. Katagiri R, Asakura K, Kobayashi S, et al. (2014) Low intake of vegetables, high intake of confectionary, and unhealthy eating habits are associated with poor sleep quality among middle-aged female Japanese workers. J Occup Health 56, 359-368.

42. Muzni K, Groeger JA, Dijk DJ, et al. (2021) Self-reported sleep quality is more closely associated with mental and physical health 
than chronotype and sleep duration in young adults: a multiinstrument analysis. J Sleep Res 30, e13152.

43. Xiao Q, Chaput JP, Olds T, et al. (2020) Sleep characteristics and health-related quality of life in 9- to 11-year-old children from 12 countries. Sleep Health 6, 4-14.

44. Fabbian F, Zucchi B, De Giorgi A, et al. (2016) Chronotype, gender and general health. Chronobiol Int 33, 863-882.

45. Merikanto I, Kronholm E, Peltonen M, et al. (2015) Circadian preference links to depression in general adult population. J Affect Disord 188, 143-148.

46. Grosso G, Micek A, Castellano S, et al. (2016) Coffee, tea, caffeine and risk of depression: a systematic review and dose-response meta-analysis of observational studies. Mol Nutr Food Res 60, 223-234.

47. Navarro AM, Abasheva D, Martinez-Gonzalez MA, et al. (2018) Coffee consumption and the risk of depression in a middle-aged cohort: the SUN project. Nutrients 10, E1333.

48. Kimura Y, Suga H, Kobayashi S, et al. (2020) Intake of coffee associated with decreased depressive symptoms among elderly Japanese women: a multi-center cross-sectional study. J Epidemiol 30, 338-344.

49. Adan A (1994) Chronotype and personality factors in the daily consumption of alcohol and psychostimulants. Addiction 89, 455-462.

50. Clark I \& Landolt HP (2017) Coffee, caffeine, and sleep: a systematic review of epidemiological studies and randomized controlled trials. Sleep Med Rev 31, 70-78.

51. Matcham F, Scott IC, Rayner L, et al. (2014) The impact of rheumatoid arthritis on quality-of-life assessed using the SF-36: a systematic review and meta-analysis. Semin Artbritis Rheum 44, 123-130.

52. Asakura T, Ishii M, Ishii K, et al. (2018) Health-related QOL of elderly patients with pulmonary $M$. avium complex disease in a university hospital. Int J Tuberc Lung Dis 22, 695-703.

53. Payne ME, Porter Starr KN, Orenduff M, et al. (2018) Quality of life and mental health in older adults with obesity and frailty: associations with a weight loss intervention. J Nutr Health Aging 22, 1259-1265.

54. Sung SA, Hyun YY, Lee KB, et al. (2018) Sleep duration and health-related quality of life in predialysis CKD. Clin J Am Soc Nephrol 13, 858-865.

55. Dai H, Mei Z, An A, et al. (2019) Association between sleep problems and health-related quality of life in Canadian adults with chronic diseases. Sleep Med 61, 26-30.

56. Allebrandt KV, Teder-Laving M, Kantermann T, et al. (2014) Chronotype and sleep duration: the influence of season of assessment. Chronobiol Int 31, 731-740.

57. Patten SB, Williams JV, Lavorato DH, et al. (2017) Seasonal variation in major depressive episode prevalence in Canada. Epidemiol Psycbiatr Sci 26, 169-176.

58. Tatsumi Y, Ishihara J, Morimoto A, et al. (2014) Seasonal differences in total antioxidant capacity intake from foods consumed by a Japanese population. Eur J Clin Nutr 68, 799-803.

59. Randler C \& Engelke J (2019) Gender differences in chronotype diminish with age: a meta-analysis based on morningness/chronotype questionnaires. Chronobiol Int 36, 888-905. 